\title{
Plants Images Classification Based on Textural Features using Combined Classifier
}

\author{
M. Z. Rashad ${ }^{1}$, B.S.el-Desouky ${ }^{2}$, and Manal S .Khawasik ${ }^{1}$ \\ Magdi_12003@yahoo.com, b_desouky@yahoo.com , and \\ manalkhawasik@yahoo.com \\ ${ }^{1}$ Dept. of Computer Science ,Faculty of Computer and Information Sciences, \\ Mansoura University ,Egypt. \\ ${ }^{2}$ Dept. Of the mathematics, Faculty of the science, \\ Mansoura University ,Egypt
}

\begin{abstract}
.
This paper introduces an approach of plant classification which is based on the characterization of texture properties. We used the combined classifier learning vector quantization. We randomly took out 30 blocks of each texture as a training set and another 30 blocks as a testing set. We found that the combined classifier method gave a high performance which is a superior than other tested methods. The experimental results indicated that our algorithm is applicable and its average correct recognition rate was $98.7 \%$.
\end{abstract}

Keywords: Plant classification, Learning vector Quantization(LVQ), Radial base function(RBF), Texture classification, Neural Networks, Texture classification.

\section{Introduction}

Image classification plays an important role in many applicable fields in our life, such as image analysis, remote sensing, and pattern recognition [9]. It can be defined as the process of sorting all the pixels in an image into a finite number of individual classes[5]. There are many types of techniques which can be used to classify and recognize different types of objects in images. For conventional statistical approaches for land cover classification, they only use the gray values of the image to detect and classify objects. They lead to misclassification due to the strictly convex boundaries. The textural features can be included for better classification but they are inconvenient for the conventional methods. On the other hand, artificial neural networks (ANNs) can handle non-convex decisions [6]. The uses of textural features help to resolve misclassification.

On the other hand, plants exist everywhere in both human environments and other places without people. Many of plants carry significant information for the human society development. The urgent situation is that many plants are at the risk of extinction, so it is very necessary to set up a database for plant protection [1-4]. We believe that the first step is to teach a computer how to classify plants. Therefore, we concentrate on the region of plant classification which plays an important role specially with people who deal with plants directly in scientific way. Our work can be used by searchers and students in the Botany field to classify different types of plants. 
International Journal of Computer Science \& Information Technology (IJCSIT) Vol 3, No 4, August 2011

\section{Literature Review}

Z. Miao et al. [12] used the evidence theory based rose classification which is based on many features of roses. This technique has a disadvantage that it's applicable to certain species. [Make the same for the rest of this paragraph.]

, X.-F. Wang, J.-X. Du, and G.-J. Zhang use a moving median center (MMC) hypersphere classifier [8]but this technique isn't fast enough specially when it's compared with approaches which are using Artificial Neural Networks(ANN), other approaches compared the similarity between features to classify plants [5] and these approach have the disadvantage of requiring preprocess work of human to enter keys manually[14], some approaches used Artificial Neural Networks(ANN) to classify opunt ia species [13] although this approach is fast but it's applicable to certain spices, J. Du, D. Huang, X. Wang, and X. Gu use shape recognition based on radial basis probabilistic neural network which is trained by orthogonal least square algorithm(OLSA) and optimized by recursive OLSA.[17] .it performs plant recognition through modified Fourier descriptors of leaf shape, many approaches which are based on leaf shape like the approach introduced by .-X. Du, D.-S. Huang, X.-F. Wang, and X. G isn't effective when the leaf shape isn't clear or destroyed the previous approach uses a modified dynamic programming algorithm for leaf shape matching .

Table 1 shows summaries of the results of other methods in order to compare between the various plant classification techniques .

Table 1 various plant classification techniques

\begin{tabular}{|c|c|c|c|c|}
\hline No. & Method & Researchers & Reference & Accuracy (\%) \\
\hline 1 & $\begin{array}{l}\text { Comparing the } \\
\text { similarity between } \\
\text { features }\end{array}$ & $\begin{array}{l}\text { Y. Ye, C. Chen, C.-T. Li, } \\
\text { H. Fu, and Z. Chi. }\end{array}$ & [5] & 71 \\
\hline 2 & PCA & $\begin{array}{l}\text { Fu, h.,Chi, Z., Feng, D., } \\
\text { and Song, J. }\end{array}$ & [4] & 80.3 \\
\hline 3 & Semi-SLLE+K-NN & Lee, C.L, Chen, and S.Y. & [25] & 85.86 \\
\hline 4 & $\mathrm{k}-\mathrm{NN}(\mathrm{k}=5)$ & $\begin{array}{l}\text { X. Gu, J. -X. Du, and X.- } \\
\text { F. Wang. }\end{array}$ & [8] & 86 \\
\hline 5 & LLE+K-NN & Shi, C., Chen, and L.H. & [10] & 86.4 \\
\hline 6 & SLLE+K-NN & Pillati,M., Viroli,C. & [11] & 88.7 \\
\hline 7 & $\begin{array}{l}\text { Wavelet transform + } \\
\text { gaussin interpolation. }\end{array}$ & $\begin{array}{l}\text { Gu, X., Du, J.-X., Wang, } \\
\text { and X. -F. }\end{array}$ & [1] & 88.9 \\
\hline 8 & $\begin{array}{l}\text { Hyper sphere } \\
\text { classifier }\end{array}$ & $\begin{array}{l}\text { Wang, X.-F., Du, J.-X, } \\
\text { Zhang, and G.-J. }\end{array}$ & [8] & 89.4 \\
\hline 9 & RBPNN & $\begin{array}{l}\text { X. Gu, J.-X.Du, and X. - } \\
\text { F. Wang. }\end{array}$ & [4] & 91 \\
\hline 10 & MMC & $\begin{array}{l}\text { J.- X.Du,X.-F. Wang, and } \\
\text { G.- J. Zhang. }\end{array}$ & [17] & 91 \\
\hline 11 & $\mathrm{k}-\mathrm{NN}(\mathrm{k}=4)$ & $\begin{array}{l}\text { J.- X.Du,X.-F. Wang, and } \\
\text { G.- J. Zhang. }\end{array}$ & [2] & 92 \\
\hline 12 & BPNN & $\begin{array}{l}\text { Fu, H., Chi, Z., Feng, D., } \\
\text { and Song, J. }\end{array}$ & [7] & 92 \\
\hline 13 & $\mathrm{PCA}+\mathrm{SVM}$ & Wu,S.G., Bao, F.S., et al. & [1] & 92 \\
\hline 14 & MLNN & $\begin{array}{l}\text { J. Du, D.Huang, X. } \\
\text { Wang, and X. Gu. }\end{array}$ & [2] & 94 \\
\hline
\end{tabular}


International Journal of Computer Science \& Information Technology (IJCSIT) Vol 3, No 4, August 2011

\begin{tabular}{|c|l|l|c|c|}
\hline 15 & $\begin{array}{l}\text { Wavelet transforms + } \\
\text { SVM }\end{array}$ & $\begin{array}{l}\text { Im, C., Nishida, H., } \\
\text { Kunii, and T.L. }\end{array}$ & {$[6]$} & 95 \\
\hline 16 & $\begin{array}{l}\text { LVQ + RBF } \\
\text { (our proposed } \\
\text { algorithm)[ ] }\end{array}$ & M. Z. Rashad & {$[28]$} & 98.7 \\
\hline
\end{tabular}

\section{Methodology}

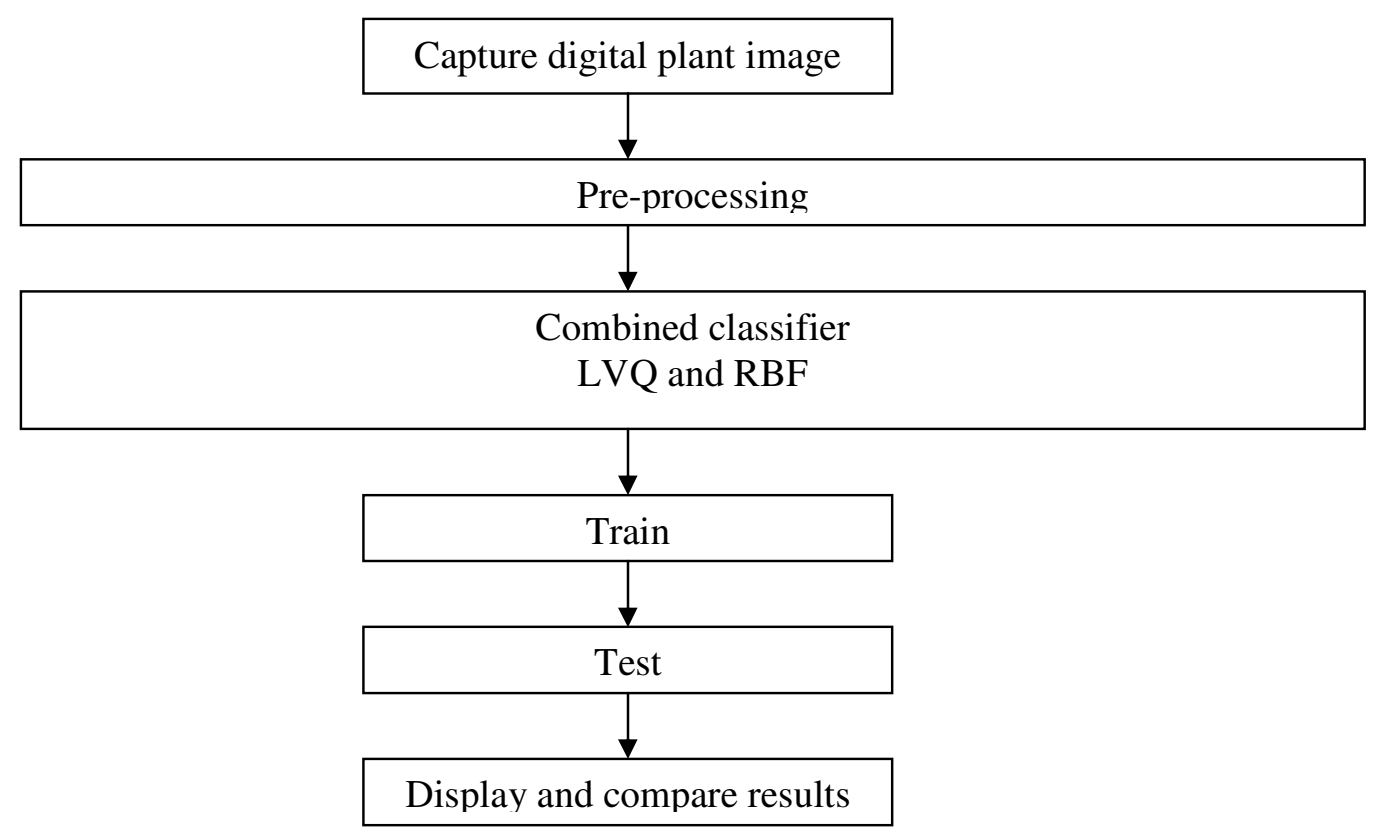

Fig. 1: The architecture of the proposed technique.

This figure is very general and you should focus on the Pre-processing and the classifier stages. Draw them in more details. They are the main contribution of your paper.

As shown in Figure 1, this image manipulate process can be divides into six steps as follows:

\subsection{Capture digital plant image}

We use a six megapixel digital camera to capture plant's images. [write camera's features] Images were taken at $(\mathrm{W} \mathrm{x} \mathrm{H})$ pixels, The image is stored in BMP format.

\subsection{Image Pre-preprocessing.}

An RGB image is firstly converted into a grayscale image.

The following Matlab code is the formula used to convert RGB image into

A grayscale image.

$$
\begin{aligned}
& \mathrm{I}=\operatorname{imread}\left({ }^{\mathrm{Path}} / / \mathrm{Im} / \mathrm{rgbim} . \mathrm{bmp}^{\prime}\right) \\
& \mathrm{J}=\operatorname{rgb} 2 \operatorname{gray}(\mathrm{I}) ; \\
& \text { Figure, imshow }(\mathrm{J})
\end{aligned}
$$

Where we save the grayscale image resulted in the matlab figure as BMP image. All plant images we use in our system are in $128 \times 128$ resolution. 


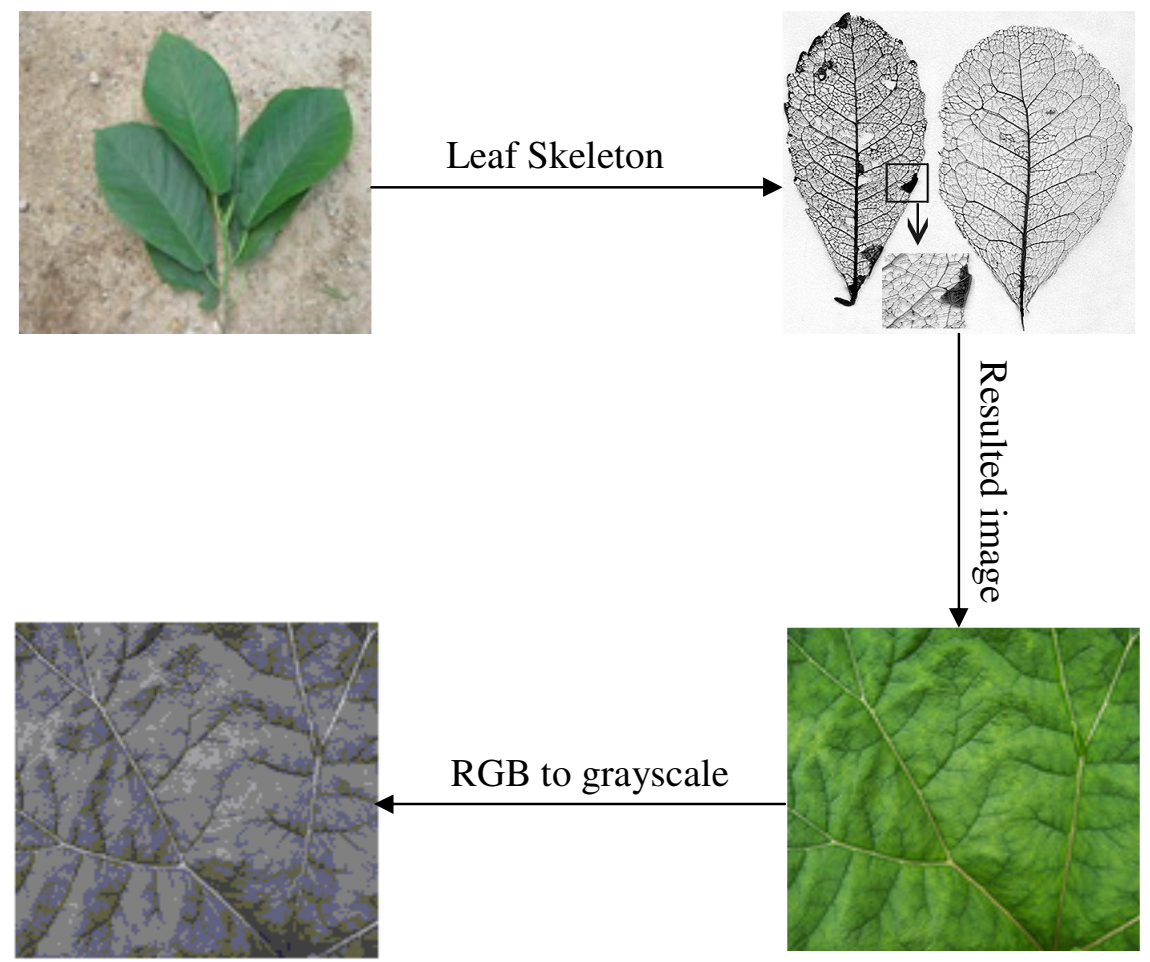

Fig. 2: A plant image pre-processing example.

\subsection{Combined classifier}

Depend on voting among several weak classifiers. And the result is the maximum vote.

\section{Results and Discussion}

To obtain the best results from our algorithm, we change the following various factors:

\section{Number of epochs:}

An epoch is the presentation of the entire training set to the neural network [9], we change the number of epochs to compare and get the best results for the system.

Where the images we use here are in $128 * 128$ resolution.

Table 2: The influence no. of epochs

\begin{tabular}{|c|c|c|}
\hline No. of epochs & Pccts & Pcctr \\
\hline 2000 & 0.97 & 0.98 \\
\hline 3000 & 0.98 & 0.985 \\
\hline 4000 & 0.987 & 0.99 \\
\hline
\end{tabular}


International Journal of Computer Science \& Information Technology (IJCSIT) Vol 3, No 4, August 2011

\section{Learning rate:}

At each training step the network computes the direction in which each bias and link value can be changed to calculate a more correct output [9]. The rate of improvement at that solution state is also known.

A learning rate is user-designated in order to determine how much the link weights and node biases can be modified based on the change direction and change rate. The higher the learning rate (max. of 1.0) the faster the network is trained. However, the network has a better chance of being trained to a local minimum solution. A local minimum is a point at which the network stabilizes on a solution which is not the most optimal global solution.

Table 3: The influence of learning rate

\begin{tabular}{|c|c|c|}
\hline Learning rate & Pccts & Pcctr \\
\hline 1.0 & & 0.98 \\
\hline 0.1 & 0.97 & 0.995 \\
\hline 0.2 & 0.98 & \\
\hline
\end{tabular}

3. Number of classifiers:

Table 4: The influence of Number of classifiers

\begin{tabular}{|c|c|c|}
\hline No. of classifiers & Pccts & Pcctr \\
\hline 4 & 0.97 & .98 \\
\hline 5 & .0 .98 & 0.987 \\
\hline 3 & 0.978 & 0.988 \\
\hline
\end{tabular}

\section{Image size:}
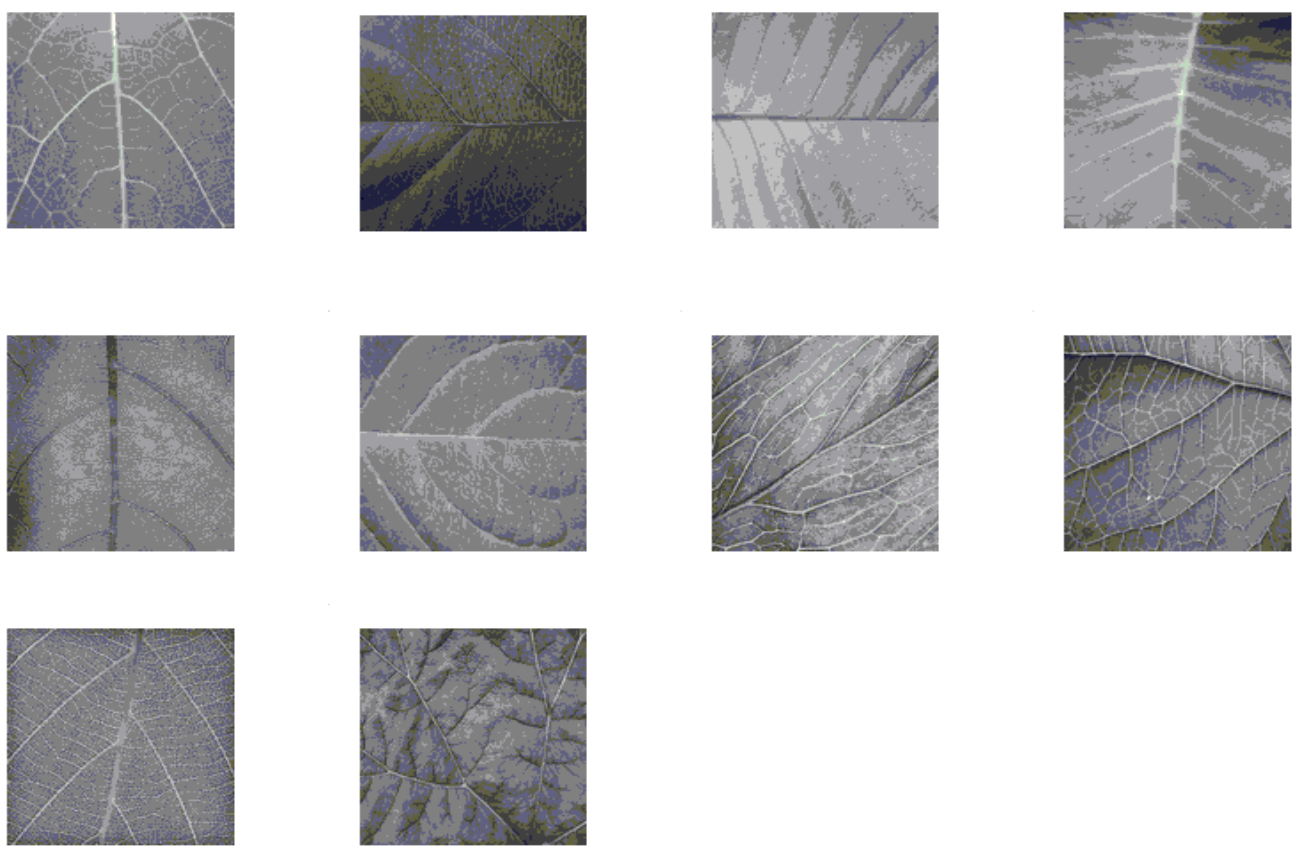

Fig. 3: The set of 10 images used for the classification 
Table 2 shows the influence of no. of epochs. If no. of epochs increases the accuracy increases. Table 3 shows the influence of learning rate. If learning rate increase the more accurate but the more time. Also table 4 shows the influence of Number of classifiers, it is the same relation.

The following figure shows various plant classification techniques. It shows that accuracy is 98.7\% compared to other systems.

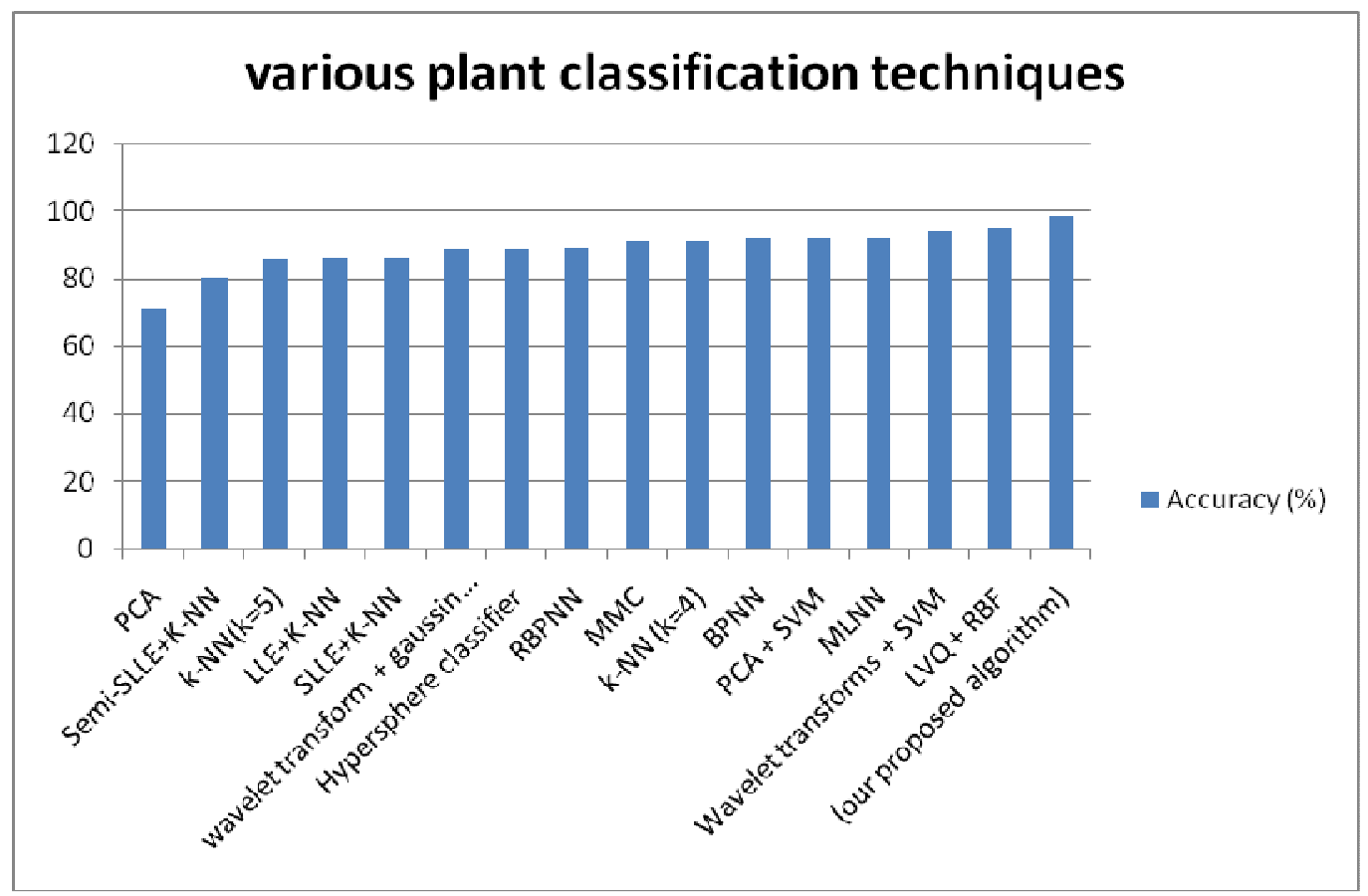

Fig 4: Various plant classification techniques

\section{Conclusion and Future Work}

Our proposed system (LVQ + RBF) is an implementation of a robust algorithm. Our accuracy is $98.7 \%$ compared to other systems.

In future, we are going to apply statistical pattern recognition methods which take noise into consideration. Our system has an advantage of its ability of classifying and recognizing the plant from a small part of the leaf without depending neither on the shape of the leaf or on its color features, since the system essentially depends on the textural features. Hence, the system is useful for the botany researchers when he wants to recognize a damaged plant, since this can be carried out depending only on a small part of the damaged plant. when the botany researcher has a damaged plant and wants to put it into its position in the classification, our system is the solution because it depends on the textural features not on the color features which is naturally changing during the seasonal succession.

\section{References}

[1] J.-X. Du, X.-F. Wang, and G.-J. Zhang, "Leaf shape based plant species recognition," Applied Mathematics and Computation, vol. 185, 2007.

[2] Du, J.X., Wang, X.F., Zhang, G.J.: "Leaf shape based plant species recognition. Applied Mathematics and Computation“"185, 883-893 (2007) 
International Journal of Computer Science \& Information Technology (IJCSIT) Vol 3, No 4, August 2011

[3] R. de Oliveira Plotze, M. Falvo, J. G. Pdua, L. C. Bernacci, M. L. C. Vieira, G. C. X. Oliveira, and O. M. Bruno, "Leaf shape analysis using the multiscale minkowski fractal dimension, a new morph metric method: a study with passifliora (passifloraceae)," Canada Journal of Botany, vol. 83, (2005)

[4] Fu, H., Chi, Z.: "Combined thresholding and neural network approach for vein pattern extraction" from ;/leaf images. In the proceedings of the IEEE Proceedings-Vision, Image and Signal Processing conference. 153(6), (2006)

[5] Y. Ye, C. Chen, C.-T. Li, H. Fu, and Z. Chi, "A computerized plant species recognition system," In proceedings of the 2004 International Symposium on Intelligent Multimedia, Video, and Speech Processing, Hong Kong, October., (2004)

[6] Im, C., Nishida, H., Kunii, T.L. D. Warren, "Automated leaf shape description for variety testing in chrysanthemums," In proceedings of the 6th IEEE International Conference Image Processing and Its Applications, (1997).

[7] Fu, H., Chi, Z., Feng, D., Song, J.: Machine learning techniques for ontology-based leaf classification. In: IEEE 2004 8th International Conference on Control, Automation, Robotics and Vision, Kunming, China (2004)

[8] X.-F. Wang, J.-X. Du, and G.-J. Zhang, "Recognition of leaf images based on shape features using a hyper sphere classifier," in Proceedings of International Conference on Intelligent Computing 2005, ser. LNCS 3644. Springer, 2005.

[9] Heraldic and Shanmugan. .Textural Features for Image Classification.. IEEE Transactions on Systems, Man and Cybernatics, vol SMC 3, no 6, November 2003, p 610.

[10] Shi, C., Chen, L.H.: Feature dimension reduction for microarray data analysis using locally linear embedding. APBC162 16, 1-7 (2004)

[11] Pillati, M., Viroli, C.: Supervised Locally Linear Embedding for Classification: An Application to Gene Expression Data Analysis. In: Proceedings of 29th Annual Conference of the German Classification Society (GfK1 2005), pp. 15-18 (2005)

[12] Miao, Z., Gandelin, M.H., Yuan, B.: An oopr-based rose variety recognition system. Engineering Applications of Artificial Intelligence 19 (2006)

[13] B. C. Heymans, J. P. Onema, and J. O. Kuti, "A neural network for opuntia leaf-form recognition," in Proceedings of IEEE International Joint Conference on Neural Networks, 1991.

[14] T. Brendel, J. Schwanke, P. Jensch, and R. Megnet, "Knowledgebased object recognition for different morphological classes of plants,” Proceedings of SPIE, vol. 2345, 1995.

[15] Alan, K.K., Philip, A., Fay, et al.: Rainfall Variability, Carbon Cycling, and Plant Species Diversity In A Mesic Grassland. Science 298, 2202-2205 (2002)

[16] J. Du, D. Huang, X. Wang, and X. Gu, "Shape recognition based on radial basis probabilistic neural network and application to plant species identification," in Proceedings of 2005 International Symposium of Neural Networks, ser. LNCS 3497. Springer, 2005.

[17] Shlens, J.: A Tutorial on Principal Component Analysis (2005), http://www.cs.cmu.edu/_elaw/papers/pca.pdf

[18] H. Demuth and M. Beale. Neural Network Toolbox. The MathWorks, Inc., Natick, Massachusetts 01760, 1998.

[19] Abbasi, S., Mokhtarian, F., Kittler, J.: Reliable Classification of Chrysanthemum Leaves through Curvature Scale Space. In: Proceeding of International Conference on Scale-Space Theory in Computer Vision, pp. 284-295 (1997)

[20] Mokhtarian, F., Abbasi, S.: Matching Shapes with Self-Intersection: Application to Leaf Classification. IEEE Transaction on Image Processing 13(5), 653-661 (2004)

[21] Saitoh, T.K.: Takeshi.: Automatic recognition of wild flowers. In: Proceedings of $15^{\text {th }}$ International Conference on Pattern Recognition (ICPR 2000), vol. 2 (2000) 
International Journal of Computer Science \& Information Technology (IJCSIT) Vol 3, No 4, August 2011

[22] Heymans, B.C., Onema, J.P., Kuti, J.O.: A neural network for opuntia leaf-form recognition.In: Proceedings of IEEE International Joint Conference on Neural Networks (1991)

[23]. Ye, Y., Chen, C., Li, C.T., Fu, H., Chi, Z.: A computerized plant species recognition system. In: Proceedings of 2004 International Symposium on Intelligent Multimedia, Video and Speech Processing, Hong Kong (October 2004)

[24] Lee, C.L., Chen, S.Y.: Classification for Leaf Images. In: 16th IPPR Conference on Computer Vision, Graphics and Image Processing, vol. 8, pp. 17-19 (2003)

[25] Hong, S.M., Simpson, B., Baranoski, G.V.G.: Interactive venationbased leaf shape modeling. Computer Animation and Virtual Worlds. 16 (2005)

[26] Nam, Y., Hwang, E.-J., Byeon, K.: ELIS: An efficient leaf image retrieval system. In: Singh, S., Singh, M., Apte, C., Perner, P. (eds.) ICAPR 2005. LNCS, vol. 3687, pp. 589- 597. Springer, Heidelberg (2005).

[27] M. Z. Rashad ,"Rotated Texture Classification Using a Combined LVQ Classifier", GESTS International Transactions on Computer Science and Engineering, vol 39, No.1, April,30,2007. 